\title{
Phospholipids and phospholipid-bound fatty acids and aldehydes of spermatozoa and seminal plasma of rhesus monkeys
}

\author{
Annabelle Darin-Bennett, I. G. White and D. D. Hoskins* \\ Department of Veterinary Physiology, University of Sydney, New South Wales 2006, Australia \\ and *Oregon Regional Primate Research Center, \\ Beaverton, Oregon 97005, U.S.A.
}

\begin{abstract}
Summary. The major components of the phospholipids of rhesus monkey spermatozoa are phosphatidyl choline $(33 \%)$, phosphatidyl ethanolamine $(25 \%)$, ethanolamine plasmalogen $(16.1 \%)$, sphingomyelin $(8.1 \%)$, choline plasmalogen $(6.9 \%)$ and cardiolipin $(4.5 \%)$. The major phospholipid-bound fatty acids are $16: 0,18: 0,18: 1$ and $22: 6$; the major fatty aldehydes are $15: 0,16: 0$ and $18: 2$. The same phospholipids are also present in the seminal plasma.
\end{abstract}

The composition of sperm phospholipids and phospholipid-bound fatty acids and aldehydes has been established in a number of species (see Darin-Bennett, Poulos \& White, 1974) including man (Poulos $\&$ White, 1973; Poulos, Darin-Bennett \& White, 1973). However, no data seem to be available on the nature of these membrane components in non-human primates. In view of the use of the monkey as a model for research in human reproduction and the possible evolutionary significance, the phospholipids of the spermatozoa and seminal plasma of the monkey were studied to determine the extent of similarity to human spermatozoa.

Ejaculates from 2 to 5 male rhesus monkeys (Macaca mulatta) were pooled, diluted with 3 volumes of $0.9 \%(\mathrm{w} / \mathrm{v}) \mathrm{NaCl}$ and centrifuged at $500 \mathrm{~g}$ for $20 \mathrm{~min}$ at room temperature $\left(20-25^{\circ} \mathrm{C}\right)$. The pellet was resuspended to $10 \mathrm{ml}$ in $0.9 \% \mathrm{NaCl}$. The sperm suspension and seminal plasma supernatant were extracted within 90 min of collection with 3.75 volumes of chloroform : methanol $(2: 1, v / v)$ and stored under liquid nitrogen at $-79^{\circ} \mathrm{C}$ during transport to the laboratory where it was kept at $-15^{\circ} \mathrm{C}$ until analysis.

The treatment of the lipid extracts and the method of analysing the phospholipids have been previously described (Darin-Bennett et al., 1973). The phospholipid-bound fatty acid and aldehyde composition were estimated by gas-liquid chromatography according to the methods of Poulos, Darin-Bennett \& White(1973).

The major components of rhesus sperm phospholipids (Table 1) are phosphatidyl choline, phosphatidyl ethanolamine and ethanolamine plasmalogen, with lesser amounts of sphingomyelin, choline plasmalogen, and cardiolipin. These components were also present in the seminal plasma but not in the same proportions. Comparison of the phospholipid composition of monkey spermatozoa and seminal plasma with that of other species such as the ram (Quinn \& White, 1967; Neill \& Masters, 1973; Darin-Bennett et al., 1973), bull (Pursel \& Graham, 1967; Poulos, Voglmayr \& White, 1973; Neill \& Masters, 1972; Darin-Bennett et al., 1973), boar (Johnson, Gerrits \& Young, 1969; DarinBennett et al., 1973), man (Poulos \& White, 1973), dog and fowl (Darin-Bennett et al., 1974), and rabbit (A. Darin-Bennett, unpublished observations) indicate clear species differences. The pattern of phospholipid composition of monkey spermatozoa would seem to resemble most closely that of the dog. Monkey spermatozoa contain higher amounts of both choline and ethanolamine plasmalogen and a lower proportion of sphingomyelin than are found in human spermatozoa.

Monkey seminal plasma phospholipids are also present in different proportions from those of human seminal plasma, the main differences being in the levels of sphingomyelin and phosphatidyl choline. Apart from the lower proportion of choline plasmalogen in monkey seminal plasma, 
Table 1. Phospholipid composition of rhesus monkey spermatozoa and seminal plasma

\begin{tabular}{|c|c|c|}
\hline Component & Spermatozoa & Seminal plasma \\
\hline Origin & $0.9 \pm 0.6$ & $0.9 \pm 0.5$ \\
\hline Sphingomyelin & $8 \cdot 1 \pm 1 \cdot 2$ & $16.9 \pm 1 \cdot 3$ \\
\hline Phosphatidyl serine & $1.6 \pm 0.7$ & $1.7 \pm 0.4$ \\
\hline Phosphatidyl inositol & $<1.0$ & $2.0 \pm 1 \cdot 1$ \\
\hline Choline plasmalogen & $6.9 \pm 1.8$ & $3.5 \pm 1.4$ \\
\hline Phosphatidyl choline* & $33.0 \pm 0.6$ & $21.8 \pm 1.6$ \\
\hline Ethanolamine plasmalogen & $16 \cdot 1 \pm 1 \cdot 1$ & $24 \cdot 0 \pm 0 \cdot 5$ \\
\hline Phosphatidyl ethanolamine $\dagger$ & $25 \cdot 0 \pm 1 \cdot 1$ & $15 \cdot 1 \pm 0.9$ \\
\hline Cardiolipin & $4.5 \pm 0.2$ & $1.3 \pm 0.4$ \\
\hline Miscellaneous lipids & $4 \cdot 0$ & $11 \cdot 3$ \\
\hline
\end{tabular}

Each result is expressed as a percentage of the total phospholipid extracted and is the mean of three analyses \pm standard error.

* The phosphatidyl choline zone may include 1-alkyl,2-acyl glycero-3-phosphorylcholine.

† The phosphatidyl ethanolamine zone may include 1-alkyl,2-acyl glycero-3-phosphoryl-ethanolamine.

the phospholipid composition bears some resemblance to that of bull seminal plasma (Pursel $\&$ Graham, 1967). It would seem, therefore, that the phospholipid composition of both the spermatozoa and the seminal plasma of the monkey differ from those of other species so far examined.

Table 2. Phospholipid-bound fatty acids of rhesus monkey spermatozoa and seminal plasma

\begin{tabular}{|c|c|c|}
\hline Fatty acid & Spermatozoa & Seminal plasma \\
\hline $14: 0$ & N.D. & $1.5 \pm 0.1$ \\
\hline $16: 0$ & $29 \cdot 2 \pm 1 \cdot 0$ & $38.2 \pm 3.8$ \\
\hline $18: 0$ & $11.2 \pm 0.9$ & $18 \cdot 3 \pm 1 \cdot 7$ \\
\hline $18: 1$ & $14.8 \pm 1.5$ & $14 \cdot 1 \pm 1 \cdot 6$ \\
\hline $18: 2$ & $3.8 \pm 0.2$ & $1.6 \pm 0.2$ \\
\hline $18: 3$ & N.D. & $4.6 \pm 0.6$ \\
\hline $20: 0$ & $3 \cdot 1 \pm 0 \cdot 1$ & $1.5 \pm 0.8$ \\
\hline $20: 4 \ddagger$ & $8.9 \pm 0.4$ & $8.9 \pm 0.6$ \\
\hline $22: 5^{*}$ & $2.8 \pm 0.8$ & N.D. \\
\hline \multirow{3}{*}{$\begin{array}{c}22: 6 \\
\text { Miscellaneous } \dagger\end{array}$} & $25.2 \pm 0.9$ & $10 \cdot 2 \pm 5 \cdot 3$ \\
\hline & $1 \cdot \overline{2}$ & $0 . \overline{7}$ \\
\hline & $(14: 0,16: 1,17: 0)$ & $(17: 0)$ \\
\hline
\end{tabular}

Each fatty acid (chain length : number of double bonds) is expressed as a percentage of the total weight of fatty acid. Mean values of three analyses are given \pm standard error. N.D., indicates that the fatty acid was either not detected or was present in amounts below $1 \%$.

* Values for this fatty acid are expressed as a percentage of total peak area.

$\uparrow$ Fatty acids identified in this fraction are indicated in parentheses.

$\ddagger$ This fraction may contain 20:3.

This, however, is not the case for the phospholipid-bound fatty acids of monkey spermatozoa (Table 2), since the composition bears a close resemblance to those of the rabbit, dog and man (Poulos, Darin-Bennett \& White, 1973; Darin-Bennett et al., 1974). The similarity to human spermatozoa is particularly striking in that the major polyunsaturated acid was docosahexaenoic acid; high levels of arachidonic acid were also present. The main saturated acid was palmitic acid, and the 18-carbon fatty acids were also prominent. The ratio of polyunsaturated : saturated fatty 
Table 3. Phospholipid-bound aldehydes of spermatozoa and seminal plasma of rhesus monkeys

\begin{tabular}{|c|c|c|}
\hline Fatty aldehyde & Spermatozoa & Seminal plasma \\
\hline $14: 0$ & $3 \cdot 2 \pm 0 \cdot 1$ & $8 \cdot 7 \pm 1 \cdot 4$ \\
\hline $15: 0 \mathrm{br}$ & $9.9 \pm 0.6$ & $9 \cdot 9 \pm 2 \cdot 6$ \\
\hline $15: 0$ & $21 \cdot 5 \pm 2 \cdot 6$ & $7 \cdot 0 \pm 2 \cdot 4$ \\
\hline $16: 0$ & $43.7 \pm 1.6$ & $25 \cdot 6 \pm 2 \cdot 5$ \\
\hline $16: 1$ & $4 \cdot 3 \pm 1 \cdot 7$ & $17 \cdot 0 \pm 6 \cdot 1$ \\
\hline $17: 0$ & N.D. & $4.4 \pm 0.9$ \\
\hline $18: 0$ & $1.4 \pm 0.4$ & $7 \cdot 8 \pm 1.0$ \\
\hline $18: 1$ & $2.6 \pm 0.7$ & $5.6 \pm 1.7$ \\
\hline $18: 2$ & $12 \cdot 7 \pm 3 \cdot 7$ & $13 \cdot 1 \pm 1 \cdot 6$ \\
\hline Miscellaneous & $\overline{0.8}$ & \\
\hline aldehydes & $(17: 0)$ & 0.0 \\
\hline
\end{tabular}

Each aldehyde (chain length : number of double bonds) is expressed as a percentage of the total peak area. Mean values for three analyses \pm standard error are given. N.D., indicates that the fatty acid was either not detected or was present in amounts below $1 \%$.

acids in monkey sperm phospholipids is 1.08 , which would probably influence the membrane reaction of monkey spermatozoa to environmental stress (Poulos, Darin-Bennett \& White, 1973; Darin-Bennett et al., 1974) and justify grouping with human, rabbit, dog and fowl spermatozoa, anticipating that monkey, like human spermatozoa, might be comparatively resistant to cold shock. The phospholipids of monkey seminal plasma have a polyunsaturated : saturated fatty acid ratio of 0.46 . Palmitic acid predominated and a high level of arachidonic acid, which may act as a precursor of prostaglandins, was present.

The aldehydes of the phospholipids of monkey spermatozoa (Table 3) are also similar to those of human, rabbit, dog and fowl and unlike those of ram, bull and boar (Poulos, Darin-Bennett \& White, 1973; Darin-Bennett et al., 1974) spermatozoa in that, although palmitaldehyde predominated, there were high levels of 18-carbon aldehydes. In monkey seminal plasma there was also a high proportion of palmitolealdehyde.

It would seem, therefore, that monkey spermatozoa are similar, although by no means identical, to human spermatozoa with respect to phospholipid-bound fatty acids and aldehydes, and are somewhat different in respect of phospholipid composition. This would indicate some differences in the structure of the membrane but the significance of this is not at present clear.

We thank Professor C. W. Emmens for his interest and advice, and W.H.O. and the Australian Research Grants Committee for financial support.

\section{References}

Darin-Bennetr, A., Poulos, A. \& White, I.G. (1973) The effect of cold-shock and freeze-thawing on the release of phospholipids by ram, bull and boar spermatozoa. Aust.J. biol. Sci. 26, 1409-1420

Darin-Bennett, A., Poulos, A. \& White, I.G. (1974) The phospholipids and phospholipid-bound fatty acids and aldehydes of dog and fowl spermatozoa. J. Reprod. Fert. 41, 471-474.

Johnson, L.A., Gerrits, R.J. \& Young, E.P. (1969) The fatty acid composition of porcine spermatozoa phospholipids. Biol. Reprod. 1, 330-334.

Neill, A.R. \& Masters, C.J. (1972) Metabolism of fatty acids by bovine spermatozoa. Biochem. J. 127, 375-385.
Neill, A.R. \& MAsters, C.J. (1973) Metabolism of fatty acids by ovine spermatozoa.J. Reprod. Fert. 34 279-287.

Poulos, A. \& White, I.G. (1973) The phospholipid composition of human spermatozoa and seminal plasma.J. Reprod. Fert. 35, 265-272.

Poulos, A., Darin-BennetT, A. \& White, I.G. (1973) The phospholipid-bound fatty acids and aldehydes of mammalian spermatozoa. Comp. Biochem. Physiol. 46, 541-549.

Poulos, A., Voglmayr, J.K. \& White, I.G. (1973) Phospholipid changes in spermatozoa during passage through the genital tract of the bull. Biochim. biophys. Acta 306, 194-202. 
Pursel, V.G. \& Graham, E.F. (1967) Phospholipids of bovine spermatozoa and seminal plasma. J. Reprod. Fert. 14, 203-211.
Quinn, P.J. \& WhtTe, I.G. (1967) Phospholipid and cholesterol control of epididymal and ejaculated ram spermatozoa and seminal plasma in relation to cold-shock. Aust. J. biol. Sci. 20, 1205-1215.

Received 13 May 1976 